International Journal of Pure and Applied Mathematics

Volume 116 No. 1 2017, 49-59

ISSN: 1311-8080 (printed version); ISSN: 1314-3395 (on-line version)

url: http://www.ijpam.eu

doi: 10.12732/ijpam.v116i1.5

\title{
APPLICATIONS OF STEIN-CHEN METHOD FOR THE PROBLEM OF COINCIDENCES
}

\author{
Chanokgan Sahatsathatsana \\ Department of Science and Mathematics \\ Kalasin University \\ 46000, THAILAND
}

\begin{abstract}
In probability theory, the problem of coincidences (matches, rencontres) was presented in the analysis of games of chance: From the top of a shuffled deck of $n$ cards which are drawn one at a time. We show how the Stein-Chen method can be applied to study The Problem of Coincidences.
\end{abstract}

AMS Subject Classification: 60G07

Key Words: Stein-Chen method, Poisson approximation, The problem of coincidences

\section{Introduction}

The problem of coincidences (matches, rencontres) originates in the game of thirteen (jeu du treize) and was proposed in 1708 by the mathematician Pierre Rémand de Montmort [5] in Essay d' Analyse sur Jeux de Hazard. We are consequently, interested in the following models:

- Model I. A box contains $n$ cards marked $1,2, \ldots, n$. and all the $n$ cards are drawn from the box one by one without replacement. We can say that a coincidence (match) occurs if the face value on a card coincides with the order in which it is drawn. For instance, if the face values of a five-card deck appear in the order 25341, then there are two matches (3 and 4).

$\begin{array}{lr}\text { Received: } & \text { January 29, } 2017 \\ \text { Revised: } & \text { June } 11,2017 \\ \text { Published: } & \text { August 29, } 2017\end{array}$

(c) 2017 Academic Publications, Ltd. url: www.acadpubl.eu 
We can construction of random variable for the obtaining the problem by the following let

$$
W_{n}=\sum_{i=1}^{n} X_{i}
$$

where $X_{i}$ are indicator variable of card numbered $i$ is at the $i^{\text {th }}$ place.

Then $i \in\{1, \ldots, n\}$. Let $W_{n}$ be the total number of matches. For $n$ beginning sufficiently large, it is logical to approximate the distribution of $W_{n}$ by Poisson distribution with mean

$$
\lambda=\mathrm{E} W_{n}=n P\left(X_{i}=1\right)=n \frac{(n-1) !}{n !}=1 .
$$

In 2006, Kanint Teerapabolarn and Kritsana Neammanee [8] gave a nonunifrom bound for approximating the distribution with parameter 1 in the form of

$$
\left|P\left(W \leq w_{0}\right)-\frac{1}{e} \sum_{k=0}^{w_{0}} \frac{1}{k !}\right| \leq \Delta\left(n, w_{0}\right)
$$

where

$$
\Delta\left(n, w_{0}\right)=\left\{\begin{array}{llc}
\frac{2}{e n} & \text { if } & w_{0}=0, \\
\frac{2\left(1-2 e^{-1}\right)}{n} & \text { if } & w_{0}=1, \\
\frac{2.08}{\left(w_{0}+1\right) n} & \text { if } & w_{0}=2,3, \ldots, n .
\end{array}\right.
$$

- Model II. A box contains $s$ sets of cards, each set consisting of $n$ cards marked $1,2, \ldots, n$. From the box $n$ cards are drawn one by one without replacement. We can say that a coincidence (match) occurs if the face value on a card coincides with the order in which it is drawn. For instance, if the face values of a six-card deck appear in the order 663557, then there are two matches (3 and 5).

The first question of Montmort's Matching problem is "What is the probability that there will be no coincidence?"

The aim of this paper is to approximate the distribution of The Problem of Coincidences (Model I, Model II) by using Stein-Chen coupling method which is introduced in Section 2. The following theorem is our main result.

Theorem 1.1. Let $W_{n}$ be the total number of matches in Model I. Then we have 
1. $\left|P\left(W_{n} \in A\right)-\operatorname{Poi}_{\lambda}(A)\right| \leq C_{\lambda, A} \frac{1}{n-1}$

2. $\left|P\left(W_{n} \in A\right)-P \operatorname{Poi}_{\lambda}(A)\right| \leq\left(1-e^{-\lambda}\right) \frac{1}{n-1}$

where $C_{\lambda, A}=\min \left\{1, \lambda, \frac{\triangle(\lambda)}{M_{A}+1}\right\}$,

$$
\triangle(\lambda)= \begin{cases}e^{\lambda}+\lambda-1 & \text { if } \lambda^{-1}\left(e^{\lambda}-1\right) \leq M_{A}, \\ 2\left(e^{\lambda}-1\right) & \text { if } \lambda^{-1}\left(e^{\lambda}-1\right)>M_{A}\end{cases}
$$

and

$$
M_{A}= \begin{cases}\max \left\{w \mid C_{w} \subseteq A\right\} & \text { if } 0 \in A, \\ \min \{w \mid w \in A\} & \text { if } 0 \notin A\end{cases}
$$

when $C_{w}=\{0,1, \ldots, w-1\}$.

Theorem 1.2. Let $\tilde{W}_{n}$ be the total number of matches in Model II. Then we have

1. $\left|P\left(\tilde{W}_{n} \in A\right)-P o i_{\lambda}(A)\right| \leq C_{\lambda, A} \frac{n s-s^{2}+s-1}{n}$.

2. $\left|P\left(\tilde{W}_{n} \in A\right)-\operatorname{Poi}_{\lambda}(A)\right| \leq\left(1-e^{-\lambda}\right) \frac{n s-s^{2}+s-1}{n}$.

Corollary 1.3. Let $\tilde{W}_{n}$ be the total number of matches in Model II, for $n=s$

1. $\left|P\left(\tilde{W}_{n} \in A\right)-\operatorname{Poi}_{\lambda}(A)\right| \leq C_{\lambda, A}$

2. $\left|P\left(\tilde{W}_{n} \in A\right)-\operatorname{Poi}_{\lambda}(A)\right| \leq 1-e^{-\lambda}$

\section{Poisson Approximation via Stein-Chen Method}

The Stein-Chen Method of Poisson Approximation provides a powerful technique for computing an error bound when approximating probabilities by a Poisson distribution.

Stein [2] introduced a new powerful technique for the obtaining the rate of convergence to standard normal distribution. Chen [4] applied Stein's idea to obtain approximation results for the Poisson distribution. Our starting point is the Stein equation for Poisson distribution, which gives,

$$
I_{A}(j)-P o i_{\lambda}(A)=\lambda g_{\lambda, A}(j+1)-j g_{\lambda, A}(j)
$$


$\lambda>0, j \in \mathbb{N} \cup\{0\}, A \subseteq \mathbb{N} \cup\{0\}$ and $I_{A}: \mathbb{N} \cup\{0\} \rightarrow \mathbb{R}$ be defined by

$$
I_{A}(w)= \begin{cases}1 & ; w \in A \\ 0 & ; w \notin A\end{cases}
$$

The solution $g_{\lambda, A}$ of (4) is the form

$$
g_{\lambda, A}(w)= \begin{cases}(w-1) ! \lambda^{-w} e^{\lambda}\left[\mathcal{P}_{\lambda}\left(I_{A \cap C_{w-1}}\right)-\mathcal{P}_{\lambda}\left(I_{A}\right) \mathcal{P}_{\lambda}\left(I_{C_{w-1}}\right)\right] & ; w \geq 1 \\ 0 & ; w=0\end{cases}
$$

where

$$
\mathcal{P}_{\lambda}\left(I_{A}\right)=e^{-\lambda} \sum_{l=0}^{\infty} I_{A}(l) \frac{\lambda^{l}}{l !}
$$

and

$$
C_{w-1}=\{0,1, \ldots, w-1\} .
$$

By substituting $j$ and $\lambda$ in (4) by any integer-valued random variable $W$ and $\lambda=\mathrm{E} W$, we have

$$
P\left(W_{n} \in A\right)-P o i_{\lambda} A=E\left(\lambda g_{\lambda, A}\left(W_{n}+1\right)\right)-E\left(W_{n} g_{\lambda, A}\left(W_{n}\right)\right) .
$$

In the case where the dependence between the instances of $X_{i}$ is global, there is an alternative approach to approximating the distribution of $W_{n}$. This approach is referred to as The Coupling approach, which was first proposed by Barbour ([1] 1982). This approach is particularly useful when it is possible to construct a random variable. $W_{n, i}$, for each a $i$ on a common probability space with $W_{n}$ such that $W_{n, i}$ is distributed as $W_{n}-X_{i}$ conditional on the event $X_{i}=1$.

There have been a number of successful applications of this method, Barbour ([1] 1982), Janson ([10] 1994), Lange ([6] 2003).

Theorem 2.1. If $W_{n}$ and $W_{n, i}$ are defined as above, $p_{i}=E\left(X_{i}\right)=P\left(X_{i}=\right.$ 1), $\lambda=E\left(W_{n}\right)$, then

$$
\left|P\left(W_{n} \in A\right)-\operatorname{Poi}_{\lambda}(A)\right| \leq\left\|g_{\lambda, A}\right\| \sum_{i=1}^{n} p_{i} E\left|W_{n}-W_{n, i}\right|
$$

where $\left\|g_{\lambda, A}\right\|:=\sup _{w}\left[g_{\lambda, A}(w+1)-g_{\lambda, A}(w)\right]$.

Many authors would like to determine a bound of $\left\|g_{\lambda, A}\right\|$. For $A \subseteq \mathbb{N} \cup\{0\}$, Chen ([4], 1975) prove that

$$
\left\|g_{\lambda, A}\right\| \leq \min \left\{1, \lambda^{-1}\right\}
$$


and Janson ([10], 1994) showed that

$$
\left\|g_{\lambda, A}\right\| \leq \lambda^{-1}\left(1-e^{-\lambda}\right)
$$

In case of non-uniform bound, Neammanee ([3], 2003) showed that

$$
\left\|g_{\lambda, A}\right\| \leq \min \left\{\frac{1}{w_{0}}, \lambda^{-1}\right\}
$$

and Teerapabolarn and Neammanee ([7], 2005) gave bound of $\left\|g_{\lambda, A}\right\|$ where $A=\left\{0,1, \ldots, w_{0}\right\}$ in the terms of

$$
\left\|g_{\lambda, A}\right\| \leq \lambda^{-1}\left(1-e^{-\lambda}\right) \min \left\{1, \frac{e^{\lambda}}{w_{0}+1}\right\} .
$$

In general case for any subset $A$ of $\{0,1, \ldots, n\}$, Santiwipanont and Teerapabolarn $([9], 2006)$ gave a bound in the form of

$$
\left\|g_{\lambda, A}\right\| \leq \lambda^{-1} \min \left\{1, \lambda, \frac{\triangle(\lambda)}{M_{A}+1}\right\}
$$

where

$$
\triangle(\lambda)= \begin{cases}e^{\lambda}+\lambda-1 & \text { if } \lambda^{-1}\left(e^{\lambda}-1\right) \leq M_{A} \\ 2\left(e^{\lambda}-1\right) & \text { if } \lambda^{-1}\left(e^{\lambda}-1\right)>M_{A}\end{cases}
$$

and

$$
M_{A}= \begin{cases}\max \left\{w \mid C_{w} \subseteq A\right\} & \text { if } 0 \in A, \\ \min \{w \mid w \in A\} & \text { if } 0 \notin A .\end{cases}
$$

The difficult part in applying Theorem 2.1 is to find $W_{n, i}$ which make $E \mid W_{n}-$ $W_{n, i} \mid$ small enough. This has not the solution in general. For the case of $X_{1}, \ldots, X_{n}$ are independent, we let $W_{n, i}=W_{n}-X_{i}$. Then $E\left|W_{n}-W_{n, i}\right|=p_{i}$ and from (6), we have

$$
\left|P\left(W_{n} \in A\right)-\operatorname{Poi}_{\lambda}(A)\right| \leq\left\|g_{\lambda, A}\right\| \sum_{i=1}^{n} p_{i}^{2} .
$$

The problem of the construction of $W_{n, i}$ is difficult in the case of dependent indicator summand.

In next section, we will use Theorem 2.1 to prove our main result by constructing the random variable $W_{n, i}$ which make $E\left|W_{n}-W_{n, i}\right|$ small. 


\section{Proof of Main Result}

Proof. Of Theorem 1.1. For each $i \in\{0,1,2, \ldots, n\}$, we define $X_{i}$ as following

$$
X_{i}= \begin{cases}1 & \text { if the } i^{\text {th }} \text { card is matched on Model I } \\ 0 & \text { otherwise. }\end{cases}
$$

Let $W_{n, i}=\sum_{i=1}^{n}, X_{i}$ be the total number of matches in Model I after dropping the card numbered $i$ which is matched , $i \in\{0,1,2, \ldots, n\}$, for each $w_{0} \in$ $\{0,1,2, \ldots, n-1\}$, we have

$$
P\left(W_{n, i}=w_{0}\right)=\frac{\left(n-1-w_{0}\right) !}{(n-1) !},
$$

and

$$
\begin{aligned}
P\left(W_{n}-X_{i}=w_{0} \mid X_{i}=1\right) & =\frac{P\left(W_{n}-X_{i}=w_{0}, X_{i}=1\right)}{P\left(X_{i}=1\right)} \\
& =\frac{P\left(W_{n}=w_{0}+1, X_{i}=1\right)}{P\left(X_{i}=1\right)} \\
& =\frac{\frac{\left(n-1-w_{0}\right) !}{n !}}{\frac{(n-1) !}{n !}} \\
& =\frac{\left(n-1-w_{0}\right) !}{(n-1) !} .
\end{aligned}
$$

Then $W_{n, i}$ has the same distribution as $W_{n}-X_{i}$ conditional on $X_{i}=1$.

In order to bound $E\left|W_{n}-W_{n, i}\right|$, we observe that

- In case $X_{i}=1$, we have the $i^{\text {th }}$ card is matched. Thus the total number of cards which are matched after dropping the card numbered $i, \quad i \in$ $\{0,1,2, \ldots, n\}$ equals to the number of matched cards minus 1 , that is

$$
W_{n, i}=W_{n}-1 .
$$

- In case $X_{i}=0$, the total number of matched cards after dropping the card numbered $i$ and we drawn them again, equals to the matched card minus the sum of number of the $j^{\text {th }}$ card, where $i \neq j$. Let 


$$
W_{n, i}=W_{n}-\sum_{j=1, i \neq j}^{n} X_{i} Y_{j}
$$

For each $j \in\{0,1,2, \ldots, n\}$, such that we define the indicator random variable $Y_{j}$ as follow:

$$
Y_{j}= \begin{cases}1 & \text { if the } j^{t h} \text { card is not matched in Model I } \\
\text { after we drawn the cards again } \\
0 \quad \begin{array}{l}
\text { otherwise }
\end{array}\end{cases}
$$

We know that

$$
E\left|W_{n}-W_{n, i}\right|=E\left(W_{n}-W_{n, i}\right)^{+}+E\left(W_{n}-W_{n, i}\right)^{-}
$$

where

$$
\left(W_{n}-W_{n, i}\right)^{+}=\max \left\{W_{n}-W_{n, i}, 0\right\}
$$

and

$$
\left(W_{n}-W_{n, i}\right)^{-}=-\min \left\{W_{n}-W_{n, i}, 0\right\}
$$

Form (9) and (10).

- In case $X_{i}=1$, we have $\left(W_{n}-W_{n, i}\right)^{+}=1$ and $\left(W_{n}-W_{n, i}\right)^{-}=0$

- In case $X_{i}=0$, we have $\left(W_{n}-W_{n, i}\right)^{+}=\sum_{i, j=1, i \neq j}^{n} X_{i} Y_{j}$ and $\left(W_{n}-\right.$ $\left.W_{n, i}\right)^{-}=0$.

Therefore,

$$
\left(W_{n}-W_{n, i}\right)^{+}=\sum_{i, j=1, i \neq j}^{n} X_{i} Y_{j}
$$

and $\left(W_{n}-W_{n, i}\right)^{-}=0$.

$$
E\left(W_{n}-W_{n, i}\right)^{+} \leq E\left\{\sum_{i, j=1, i \neq j}^{n} X_{i} Y_{j}\right\}
$$




$$
\begin{aligned}
& =\sum_{i, j=1, i \neq j}^{n} E\left\{X_{i} Y_{j}\right\} \\
& =\sum_{i, j=1, i \neq j}^{n} P\left(X_{i}=1, Y_{j}=1\right) \\
& =\sum_{i, j=1, i \neq j}^{n} P\left(X_{i}=1\right) P\left(Y_{j}=1\right) \\
& =\sum_{i, j=1, i \neq j}^{n} \frac{1}{n}\left(1-\frac{1}{n-1}\right) \\
& =\frac{n-2}{(n)(n-1)} \\
& \leq \frac{1}{n}
\end{aligned}
$$

Hence, by (6), (7), (8) and (11), we have

$$
\left|P\left(W_{n} \in A\right)-\operatorname{Poi}_{\lambda}(A)\right| \leq C_{\lambda, A} \frac{1}{n} .
$$

And

$$
\left|P\left(W_{n} \in A\right)-P \operatorname{Poi}_{\lambda}(A)\right| \leq\left(1-e^{-\lambda}\right) \frac{1}{n}
$$

Proof. Of Theorem 1.2. We defined $\tilde{X}_{i}$ are indicator variable of card numbered $i$ is at the $i^{\text {th }}$ place in Model II, $i \in\{0,1,2, \ldots, n\}$. So

$$
P\left(\tilde{X}_{i}=1\right)=\frac{\frac{(n s-1) !}{(s !)^{n-1}(s-1) !}}{\frac{(n s) !}{(s !)^{n}}}=\frac{1}{n} .
$$

Let $\tilde{W}_{n, i}$ be the total number of matches in Model II by dropping the card numbered $i$ which is matched, $i \in\{0,1,2, \ldots, n\}$. For $w_{0} \in\{0,1,2, \ldots, n-1\}$, that is

$$
P\left(\tilde{W}_{n, i}=w_{0}\right)=\frac{\frac{\left(n s-1-w_{0}\right) !}{(s !)^{n-1-w_{0}\left(s-1-w_{0}\right) !}}}{\frac{(n s) !}{(s !)^{n}}} .
$$


And

$$
\begin{aligned}
P\left(\tilde{W}_{n}-\tilde{X}_{i}=w_{0} \mid \tilde{X}_{i}=1\right) & =\frac{P\left(\tilde{W}_{n}-\tilde{X}_{i}=w_{0}, \tilde{X}_{i}=1\right)}{P\left(\tilde{X}_{i}=1\right)} \\
& =\frac{P\left(\tilde{W}_{n}=w_{0}+1, \tilde{X}_{i}=1\right)}{P\left(\tilde{X}_{i}=1\right)} \\
& =\frac{\frac{\left(n s-\left(w_{0}+1\right)\right) !}{(s !)^{n-\left(w_{0}+1\right)}\left(s-\left(w_{0}+1\right)\right) !}}{\frac{(n s) !}{(s !)^{n}}} \\
& =\frac{\frac{\left(n s-1-w_{0}\right) !}{(s !)^{n-1-w_{0}\left(s-1-w_{0}\right) !}}}{\frac{(n s) !}{(s !)^{n}}} .
\end{aligned}
$$

It is clear that $\tilde{W}_{n, i}$ has the same distribution as $\tilde{W}_{n}-\tilde{X}_{i}$ conditional on $\tilde{X}_{i}=1$.

In order to bound $E\left|\tilde{W}_{n}-\tilde{W}_{n, i}\right|$, we observe that

- In case $\tilde{X}_{i}=1$, we have

$$
\tilde{W}_{n, i}=\tilde{W}_{n}-1
$$

- In case $\tilde{X}_{i}=0$, we have

$$
\tilde{W}_{n, i}=\tilde{W}_{n}-\sum_{j=1, i \neq j}^{n s} \tilde{X}_{i} \tilde{Y}_{j}
$$

For each $j \in\{0,1,2, \ldots, n\}, j \neq i$ such that we define the indicator random variable $Y_{j}$ as follow:

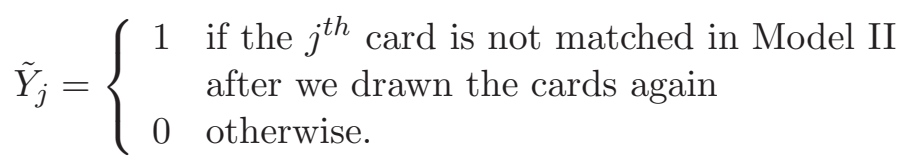

We know that

$$
E\left|\tilde{W}_{n}-\tilde{W}_{n, i}\right|=E\left(\tilde{W}_{n}-\tilde{W}_{n, i}\right)^{+}+E\left(\tilde{W}_{n}-\tilde{W}_{n, i}\right)^{-}
$$

And (12) and (13). 
- In case $\tilde{X}_{i}=1$ we have $\left(\tilde{W}_{n}-\tilde{W}_{n, i}\right)^{+}=1$ and $\left(\tilde{W}_{n}-\tilde{W}_{n, i}\right)^{-}=0$

- In case $\tilde{X}_{i}=0$ we have $\left(\tilde{W}_{n}-\tilde{W}_{n, i}\right)^{+}=\sum_{j=1, i \neq j}^{n s} \tilde{X}_{i} \tilde{Y}_{j}$ and $\left(\tilde{W}_{n}-\tilde{W}_{n, i}\right)^{-}=$ 0 .

Therefore, $\left(\tilde{W}_{n}-\tilde{W}_{n, i}\right)^{+}=\sum_{j=1, i \neq j} \tilde{X}_{i} \tilde{Y}_{j}$ and $\left(\tilde{W}_{n}-\tilde{W}_{n, i}\right)^{-}=0$.

$$
\begin{aligned}
E\left(\tilde{W}_{n}-\tilde{W}_{n, i}\right)^{+} & \leq E\left\{\sum_{j=1, i \neq j}^{n s} \tilde{X}_{i} \tilde{Y}_{j}\right\} \\
& =\sum_{j=1, i \neq j}^{n s} E\left\{\tilde{X}_{i} \tilde{Y}_{j}\right\} \\
& =\sum_{j=1, i \neq j}^{n s} P\left(\tilde{X}_{i}=1, \tilde{Y}_{j}=1\right) \\
& =\sum_{j=1, i \neq j}^{n s} P\left(\tilde{X}_{i}=1\right) P\left(\tilde{Y}_{j}=1\right) \\
& =\sum_{j=1, i \neq j}^{n s} \frac{1}{n}\left(1-\frac{(n s-2) !}{\frac{(n s-1) !}{(s-2(s-2) !}}\right) \\
& =\frac{n s-s^{2}+s-1}{n}
\end{aligned}
$$

Hence, by (6), (7), (8) and (14), we have

$$
\left|P\left(\tilde{W}_{n} \in A\right)-P \operatorname{Po}_{\lambda}(A)\right| \leq C_{\lambda, A} \frac{n s-s^{2}+s-1}{n} .
$$

And

$$
\left|P\left(\tilde{W}_{n} \in A\right)-P \operatorname{Poi}_{\lambda}(A)\right| \leq\left(1-e^{-\lambda}\right) \frac{n s-s^{2}+s-1}{n} .
$$

Proof. Of Corollary 1.3. Suppose that $n=s$, we have

$$
\begin{aligned}
E\left(\tilde{W}_{n}-\tilde{W}_{n, i}\right)^{+} & \leq \frac{n s-s^{2}+s-1}{n} \\
& \leq 1
\end{aligned}
$$

Hence, by (6), (7), (8) and (15), the proof is completed 


\section{Acknowledgements}

The author thanks the anonymous referees for their valuable suggestions and constructive criticism which improved the presentation of the paper.

\section{References}

[1] A.D. Barbour, Poisson Convergence and Random Graphs, Mathematical Proceedings of the Cambridge Philosophical Society. 92. No. 02. Cambridge University Press, 1982.

[2] C.M. Stein, A bound for the error in the normal approximation to the distribution of a sum of dependent random variables, In: Proceedings of the Sixth Berkeley Symposium on Mathematical Statistics and Probability, Volume 2: Probability Theory. The Regents of the University of California, 1972.

[3] K. Neammanee, Pointwise approximation of Poisson binomial by Poisson distribution, Stochastic Modelling and Applications, 6 (2003), 20-26.

[4] L.H.Y. Chen, Poisson approximation for dependent trials, The Annals of Probability (1975), 534-545.

[5] P.R. de Montmort, Essay D'analyse sur les Jeux de Hazard, Chez Jacque Quillau, imprimeur-jur-libraire de l'Universit, rue Galande, 1713.

[6] K. Lange, Applied Probability, Springer Science and Business Media, 2010.

[7] K. Teerapabolarn and K. Neammanee, Poisson approximation for sums of dependent Bernoulli random variables, Acta Mathematica Academiae Paedagogicae Nyiregyhaziensis, 22 (2006), 87-99.

[8] K. Teerapabolarn and K. Neammanee, A non-uniform bound on matching problem, Kyungpook Math., 46 (2006), 489-496.

[9] T. Santiwipanont and K. Teerapabolarn, Two formulas of non-uniform bounds on Poisson approximation for dependent indicators, Thai Journal of Mathematics, 1 (2006), 15-39.

[10] S. Janson, Coupling and Poisson approximation, Acta Applicandae Mathematica, 34, No. 1-2 (1994), 7-15. 
